\title{
Study on the Application of Information Intelligent Farm
}

\author{
Xing Chen, Linpeng Zhai \\ Water and energy and Power Engineering College, Yangzhou University, Yangzhou Jiangsu, 225127
}

\begin{abstract}
In order to improve the production and the management level of modern farm, it is the inevitable way of intelligent farm to realize the scientific decision and management by using the information technology. In this paper, we analyze an intelligent farm of the overall framework, the structure of hardware and the software application platform of constructing from the perspective of existing information technology. And the prospects and construction measures of information technology in the intelligent farm are described.
\end{abstract}

Index Terms - intelligent farm, information technology, management mode

\section{Summary}

Along with the agricultural development mode change in our country, especially the increase of the rural land circulation speed,which promote the development of modern agriculture and the scale of operation, accelerate the development of a lot of large-scale modern farm,the construction of Agriculture Park, at the same time, promote the continuous optimization of the structure,develop the function of industrial, grow the main business, improving infrastructure and cause science and technology achievements to appear gradually. But agricultural production still remains in the traditional management mode. In the aspect of promoting agricultural development mode change, promoting agricultural economic transformation and upgrading and achieving the efficient ecological agriculture and so on still faces many challenges [1]. So we need to improve management model and the efficiency of agricultural production by using modern information technology.

The application of information technology can better control the crops growth environment which can meet the growing demand of crops, and then improve the yield and quality of crops.It is beneficial to achieve the high and stable yield of crops, Improve the land output rate, improve the ability to withstand natural disasters and it also has important practical significance of promoting the sustainable development of agriculture.

\section{Intelligent Farm}

The so-called "intelligent farm", is making full use of the application of modern information technology to integrate the application of computer and network technology, the technology of the Internet of things, the video monitoring technology, 3S technology, wireless communication technology, integrate the decision support system of knowledge and wisdom of agricultural expert, realize the intelligent management of remote monitoring of farm visualization, remote control, disaster warning and so on [2] .
The intelligent farm is the basic unit of the development of modern agriculture, it base on each sensor node of farm production site (environmental temperature, humidity, crop growth and plant diseases and insect pests monitoring etc.), transmit to the control center by the wireless network, realize the intelligent perception, intelligent early warning, intelligent decision, intelligent analysis of the farm production environment, and then provide accurate values, visual management, intelligent decision making for farm producted.

\section{Overall Framework of the System}

The construction of intelligent farm information built up can be divided into information perception layer, transport layer and application layer information, as shown in Figure 1.

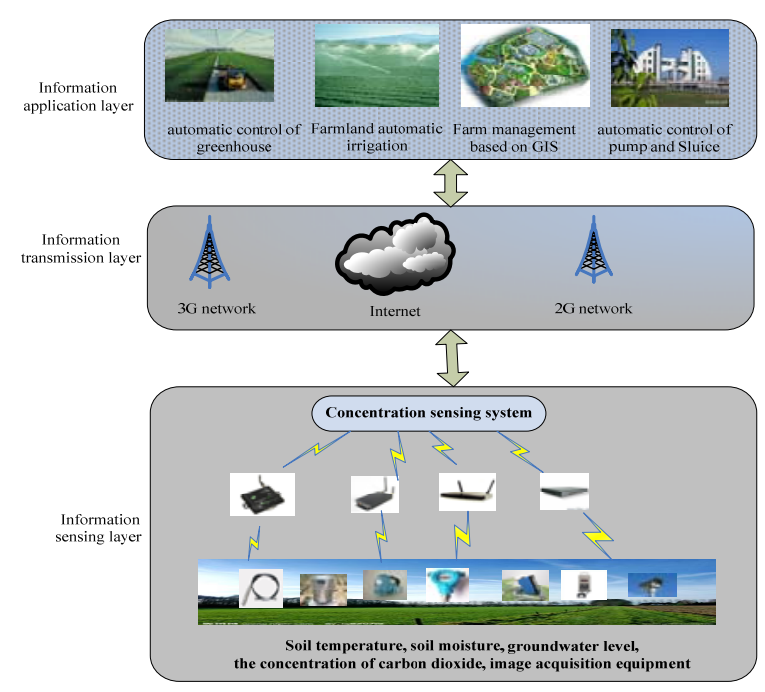

Figure 1 Structure of modern farm system

Information sensing layer at the lowest layer of the three layer architecture, it Includes two aspects of data acquisition and data transmission, collects environment and crop information by a variety of sensors, cameras, and then transmit to the gateway by short distance wireless or wired transmission technology and other data information in a variety of communication protocols [3]-[5]. Meanwhile, control commands from upper lower are transmit to the control device by the gateway, remotely control the various control equipment.

Information transmission layer connects the lower gateway equipment by the Internet, 2G, GPRS and 3G,and merge into each other, achieve more efficient, reliable, convenient, cheap and safe data transmission.It provides on-site data for the upper application layer, at the same time, transmits the expert decision-making data to the lower layer,and realize the control of field equipment. 
Information application layer is the core of the intelligent farm management system, it realizes the data collected from the perception layer to be analyzed and processed by this system, intelligent control and management of the farm production site, realizes the automation and intelligent farm, provides decision support for farm producted.

\section{Hardware System}

The hardware system is the foundation of the intelligent farm management, the design and selection should consider a few principles: (1) Practicability, which is able to adapt to the needs of measurement and management of a farm, with high reliability and operability; (2) Progressiveness, intelligent farm is the direction of agricultural development under the condition of information at present,which needs to have the appropriate advance; (3) Economy, for a farm,it is necessary to set the information decision support system, but we should consider the input and output ratio.We can take the action of uniform design,carry out step by step.

Considering the above factors, the hardware structure of the inelligent farm is as shown in Figure 2

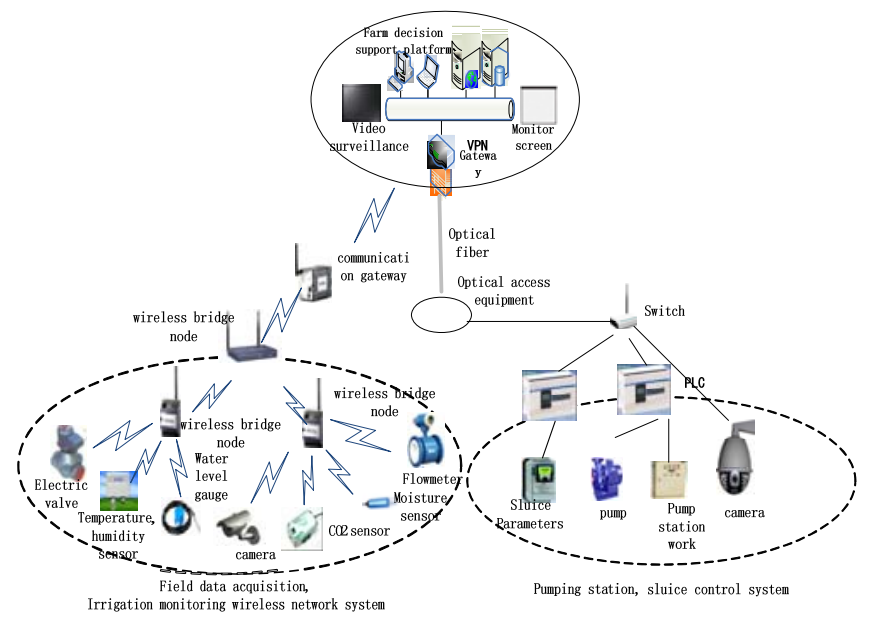

Figure 2 Schematic diagram of the hardware structure of the intelligent farm

\subsection{Data acquisition of field conditions, irrigation monitoring wireless network system}

The data acquisition system of farm field, mainly through various sensors deployed in the field, conducting real-time monitoring and digital processing on the soil temperature during crop growth, soil moisture, air temperature of the field, air humidity, solar radiation, wind, wind direction and other meteorological factors and the trend of the crop growth, and transmitting to focus point switch through wireless gateway, and transmitting to integrated management platform through the network [6]-[7].

Irrigation monitoring system are mainly composed of flow monitoring, gate control and video surveillance system. These devices are dispersed in farm field, connected by the wireless network, transmitting various types of monitoring information to the integrated management platform through the wireless gateway and the communications gateway.

By the dispersing various types of data acquisition and monitoring equipments, it can meet the various indicators' requirements of plant growth about environment and nutrient timely and accurately and conduct real-time monitoring and digital processing on the trend of the growth to guide the disaster prevention and reduction of agricultural production and production management.

\subsection{Gate and station monitoring system}

The field gate and station monitoring system have the automation controlling system of irrigation and drainage pumping station, gate monitoring system and video surveillance of the two systems. These systems set up various types of data acquisition and operation control units, transport kinds of information to the integrated management platform through the transmission network and realize the remote monitoring of the pumping station and gate.

\section{Software System}

The software system of intelligent farm includes database technology, MIS(management information system), DSS (decision support system), ES (expert system), GPS, GIS (geographic information system), RS(remote system) and so on, realize the agricultural products from the selection, breeding to the production management, subscription sales, logistics and distribution, quality and safety traceability's production, supply, sales of the whole process' efficient perception and control, and promote the transformation of traditional farm to intelligent farm. It covers farm planning, production, distribution and other aspects. It mainly complete the production, organization and management of the whole farm. The structure of the software system is shown in Figure 3.

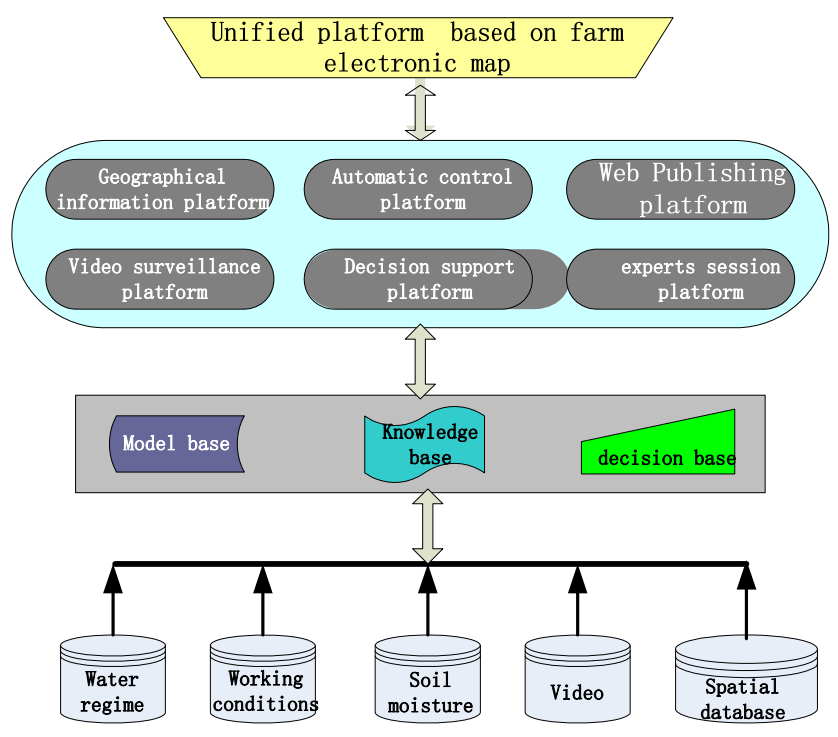

Figure 3 The software system structure of intelligent farm

The whole software system is divided into three layers. The lowest layer is the storage layer of kinds of data processing and decision-database processing, storing the collected data from the 
field, agricultural expert knowledge, decision programming model, farm spatial information, video images and so on from the acquisition equipments in the field. The second layer is a software support platform of system processing, composed of automatic control platform, geographic information processing platform, Web information processing platform, decision support platform, expert sessions platform and video surveillance platform, is the core of the software processing in intelligent farm. The third layer is based on a unified platform of geographic information system; the user interface of the whole system is based on the farm electronic map, combined with the Web application forming system interface.

\section{The Prospect of Application}

Intelligent farm IT help farm managers timely access to various production and management information. Using these information to manage field scientifically can reach the aim of increasing production, improving quality, reducing production costs, promoting circulation of agricultural products and achieving better economic benefits. It can also solve the problem of lack of agricultural technical personnel and popularize science and technology effectively.

In order to promote the widely use of IT in intelligent farm, firstly, government should give policy support; guiding and nurturing are the most import. Because the investment in IT is still relatively large, some preferential policies that are different from other industries should be given in terms of taxation and financing. Secondly, with the deepening of urbanization reform, the field managers need to manage large areas of farmland and improve agricultural productivity by means of information. In addition, participation in joint construction and development of information technology companies should their ideas and unify technical standards, reduce barriers, reduce the costs of the construction of farm information.

\section{Conclusions}

Agricultural production need to develop, stay in the traditional scale、 production and management mode, which is unable to adapt to the demands of modern agricultural development,we must follow the road of the scale and modernization. The production, management and operation of the difficulty must increase because of the increasing scale.So we must use information technology to improve the production, management and operation level.With the development of the Internet, mobile Internet, wireless sensor networks, cloud communication, cloud computing, Internet of things,applying these techniques to the intelligent farm will greatly promote the precision and intelligent management of the process of production, processing, circulation, sales of the farm.

\section{References}

[1] Jiansong Yao, Yunlong Zhong,etc. IOT technology application research of modern agriculture in Jiaxing City. Agricultural equipment technology, 2012, 28(2):4-7.

[2] Shanshan Fan,Zhong Li,etc.IOT application in intelligent agriculture. Computer CD software and application, 2013(13): 41-42

[3] Zhaojiang Xie, Wangrong Liu, etc.Relying on IOT technology to promote agricultural information. Anhui agricultural science, 2011, 39(36): 22812-22822.

[4] Sichuan Hongyu KIC information technology limited liability company. Intelligent farm solutions.IOT world, 2013.5.13.

[5] Lixin Lv, Wei Wang, Tianran Bu. Precision agriculture environment monitoring system design based on wireless sensor network. Journal of Jiangsu University, 2009, 30(6):541-544.

[6] Zhujun Liang, Li Wu. Environment monitoring technology application in facility agriculture. Anhui agricultural science, 2009, 37(16): 7672-7673, 7753.

[7] Kim Yunseop, Evans R G,Iversen W M.Remote sensing and control of an irrigation system using a distributed wireless sensor network. Ieee Transactions On Instrumentation And Measurement, 2008, 57(7): 1379-1387.

[8] Camili A, Cugnasca C E,Saraiva A M, et al. From wirless sensors to field mapping: Anatomy of an application for precision agriculture. Computers and Electronics in Agriculture, 2007, 5(8):25-36. 